\title{
Demand Characteristics of a Questionnaire Used to Assess Motion Sickness in a Virtual Environment
}

\author{
Sean D. Young ${ }^{1}$, Bernard D. Adelstein ${ }^{2}$, Stephen R. Ellis ${ }^{2}$ \\ ${ }^{1}$ Stanford University and the San Jose State University Foundation \\ Palo Alto, California, U.S.A \\ ${ }^{2}$ NASA Ames Research Center \\ Moffett Field, California, 94035-1000 U.S.A. \\ sellis@mail.arc.nasa.gov
}

\begin{abstract}
The experience of motion sickness in a virtual environment may be measured through pre- and post-experiment self-reported questionnaires such as the Simulator Sickness Questionnaire (SSQ). Although research provides converging evidence that users of virtual environments can experience motion sickness, there have been no controlled studies to determine to what extent the user's subjective response is a demand characteristic resulting from pre- and post-test measures. In this study, subjects were given either SSQ's both pre and post virtual environment immersion, or only post immersion. This technique was used to test for contrast effects due to demand characteristics in which administration of the questionnaire itself suggests to the participant that the virtual environment may produce motion sickness. Results indicate that reports of motion sickness after immersion in a virtual environment are much greater when both pre and post questionnaires are given than when only a post test questionnaire is used. The implications for assessments of motion sickness in virtual environments are discussed.
\end{abstract}

CR Categories and Subject Descriptors: Motion sickness, cyber sickness, demand characteristics, user study

Keywords: manual control, three dimensional tracking, placebo effect, virtual environment

\section{Introduction}

"Even the dullest college student is aware that some change is expected, particularly if the test is in some obvious way related to the treatment" [1].

\subsection{Experimental demand characteristics}

In 1891 a horse named Clever Hans began amazing people by apparently solving simple mathematics problems. Spectators would come from all over the world to witness the brilliant horse perform. When his owner would ask him a question, for example, to calculate the sum of two and two, Clever Hans would mystify the audience by stomping four times with his hoof. Years later, researchers discovered that Clever Hans was not a mathematician at all. Instead, he was just sensitive to environmental cues and would stomp out the answer until his owner or others in the audience who knew the answer to the problem subtly and unconsciously cued him to respond [2]. Clever Hans's mathematical responses were influenced (and confounded) by demand characteristics and experimenter expectancy effects.

A demand characteristic is the "sum total of cues that convey an experimental hypothesis to subjects and influence their behaviour" [1]. The most basic example of a demand characteristic is an experimental procedure that involves a pre-measure, immediately followed by an experimental treatment, which in turn is followed by a post-measure using the same test as did the premeasure. The post-measure is thereby influenced by contrast effects, or an implicit understanding that the experimental treatment should make the post-measure differ from the pre-measure. Experimenter expectancy effects are a type of experimenter-induced demand characteristic whereby an authoritative person's expectations come to evoke the expected behaviour [3]. While conducting research, it is important to create experiments with minimal demand characteristics to remove the possibility that the subjects are responding to the experimenter's cues. A major problem with demand characteristics is that they bias the results in favour of the experimental hypothesis, inaccurately confirming the researcher's beliefs about the experiment [4].

Experimenter demand has been found to affect research in a variety of domains including surgical procedures, physical manipulations, prescribed diets, exercises, and other regimens [5],[6]; weakness, nausea, rashes, and pain, fainting, vomiting, diarrhoea, changes in blood flow, and detrimental respiratory effects [7],[8]. Questionnaire data is particularly vulnerable to these effects since the data is less objective. Self-reported questionnaire data can lead to response bias [9] as a result of experimenter demand and self-presentation [10]. That is, the participant's self-report may be influenced by the experimenter's cues and desire to confirm his or her hypothesis.

\subsection{Motion Sickness in Virtual Environments}

Virtual environments are interactive computer images that employ a head-mounted display (HMD) to convey information to the user. Virtual environment users are immersed in a synthetic space of virtual images enhanced by special processing and nonvisual display modalities [11]. Research on virtual environments has provided converging evidence that being placed in a virtual environment with an HMD can lead to motion sickness [12]. For example, Kennedy, Fowlkes, and Lilienthal [13], have shown that following exposure to virtual environments and simulators, 
up to $30 \%$ of exposures result in reported nausea, up to $40 \%$ of exposures result in reported eyestrain, as well as the experience of numerous other symptoms of motion sickness including drowsiness, salivation, sweating, headache, and dizziness/vertigo, and loss of postural stability. Although the prevalence of motion sickness in virtual environments appears greater than in flight simulators [14], results are generally based upon survey and selfreported measures rather than controlled experiments [15],[16].

It is therefore possible that the user of a virtual environment may not actually be experiencing motion sickness but may just be reporting it, in part due to the demand characteristics of the questionnaire technique. The aim of the present study is to determine the extent to which users' reports of motion sickness after experience within virtual environments might be due to the demand characteristics of a questionnaire measurement technique.

The heightened likelihood of experiencing motion sickness in a virtual environment may be a result of the methods for gathering motion sickness data. Motion sickness data may be measured, for example, with either a Pensacola Motion Sickness Questionnaire (MSQ) or a simulator sickness questionnaire (SSQ) [17]. The SSQ was created and is commonly used specifically for measuring motion sickness in virtual environments because the MSQ does not provide information about the multiple separable dimensions of motion sickness [18] The SSQ contains a symptom rating list consisting of 16 symptoms which are self-reported by the participant on a 4 -point scale $(0=$ none, $1=$ slight, $2=$ moderate, $3=$ severe). These ratings form the basis of three subscale scores (Nausea, Oculomotor Discomfort, Disorientation), and combine to form a Total Severity score. Examples of the symptoms in the questionnaire are: general discomfort, sweating, nausea, difficulty concentrating, fatigue, headache, eyestrain, dizziness, and vertigo.

The Total Severity score takes a composite of all of the symptoms and calculates the overall extent of symptom severity. The SSQ subscale scores can provide more specific diagnostic information as to nature of the resulting sense of sickness. We will employ the SSQ and, in particular, its Nausea subscale as our measure of motion sickness, using these scores somewhat generically since our computer simulation does not generate physical motion.

Since the SSQ is administered as a pre-and post-test measure [17], [19], it may be influenced by demand characteristics. This kind of problem with pre/post-testing in virtual environments has been discussed previously by Nichols, Cobb and Wilson [20]. One specific problem with the pre/post test use is that seeing the list of symptoms beforehand may increase the user's sensitivity to those symptoms and provide information that motion sickness is common, or common enough to be worth measuring. In other words, the user, seeing the SSQ prior to immersion in the virtual environment, may be more aware of physiological changes than he or she otherwise would. Additionally, upon receiving a postquestionnaire, the user may remember that these symptoms were recorded on the pre-questionnaire and may perceive that the "appropriate answer" is to report differences between pre and post measures. Any differences on the questionnaire would then be due to demand characteristics and contrast effects. Accordingly, our directional hypothesis for this experiment is that subjects who are exposed to a pre-questionnaire assessing simulator sickness will be more likely to report sickness in the virtual environment than those who are exposed to a questionnaire only after immersion in the environment.

\section{Methods.}

Thirty subjects between 20 to 45 years old ( 20 men and 10 women) and inexperienced in the use of virtual environments were recruited and brought to the Advanced Displays and Spatial Perception Laboratory at Ames Research Center. Subjects were randomly assigned to a pre- and post-test group or a post-test only group. Subjects in the pre/post condition were given an SSQ (see [17] for measures) both prior to and immediately after immersion in a virtual environment. Subjects in the post condition received the SSQ only immediately after immersion.

\subsection{Technical details of virtual environment simulation}

Subjects wore a Virtual Research V8 TFT-LCD stereoscopic HMD to which was affixed the receiver of a spatially calibrated Polhemus Fastrak position and orientation sensor [21]. The HMD is specified as having a $48^{\circ} \mathrm{X} 32^{\circ}$ (horizontal $\mathrm{X}$ vertical) field of view (FOV) per eye at VGA $(640 \times 480$ triads $)$ resolution. The HMD and Fastrak were interfaced to a dual-Xeon $(2.4 \mathrm{GHz})$ Dell Precision 530 workstation, operating under Windows 2000, and equipped with a commodity NVidia GeForce FX-5900 graphics card.

The C++ software application that both generated the stereoscopically rendered virtual environment and controlled the progress of the experiment was built on a WorldTookKit framework written in-house. Standalone tracker software (AuAST from AuSim, Inc., Mountain View, CA), running as a background Windows service, deposited streaming (i.e., continuous rather than polled) Fastrak measurements into shared memory for retrieval as needed by the virtual environment application. Internal timing [22] is used to adjust the reading of the Fastrak measurements to minimize the latency between the reading of head position signal and the video display to the HMD.

This combination of shared memory data transfer and multiprocess synchronization enabled the $\sim 800$ polygon experiment virtual environment illustrated in Figure 1 to operate at a pre-set, uniform $25 \mathrm{~ms}$ internal latency-i.e., the interval from the instant of the Fastrak measurement until the first pixel arrives at the HMD. The virtual environment update rate was fixed at $60 \mathrm{~Hz}$ by the refresh rate of the slowest component in our virtual environment system, the V8 HMD.

\subsection{Experimental task}

In the virtual environment, subjects played a virtual balloon popping "game" that consisted of 60 trials in which they positioned a $3 \mathrm{D}$, virtual ball cursor $(2 \mathrm{~cm}$ diameter) by moving their dominant hand. They were told to move their cursor toward spherical targets located randomly but visibly in front of them within arm's reach. The targets often required about a half step of motion to allow the participant to make contact with them. The subject's task was to move their cursor through virtual space to contact the virtual "balloon" which disappeared ("popped") when contact was made. The targets varied randomly in diameter between 2 and $35 \mathrm{~cm}$ and were presented so that the participant did not have to search extensively for them at the beginning of each targeting movement. All targeting movements began from a position adjusted to be directly in front and within arm's reach for each subject. 


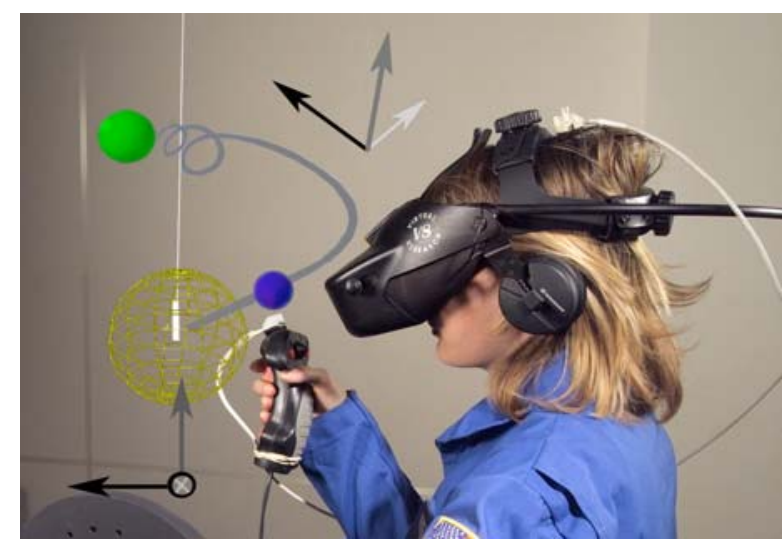

Figure 1. Schematic illustration of the subject's task. The subject moved a 3D cursor shown as virtual ball just above the tracked handle. The cursor was moved from a starting point within the larger wire-freame sphere just at the end of the hanging white plumb bob to contact a the medium-sized ball-like target shown in the upper right. The curved path shows a typical corkscrew structure that particpants tended to exhibit when experiencing a coordinate rotation like that illustrated between the coordinate frame in the lower left and that in the upper center of the figure. The plumb bob could be felt to find the initial starting point of the motion. Neither the coordinate frame icons nor the path trace were visible to the subject. The starting sphere did not remain visible once the targeting motion was started.

The display of targeting movements was affected by threedimensional rotational control misalignments, such that when participants attempted to move their cursor toward the target they would receive visual feedback that they were moving on a different course. They therefore had to constantly monitor and continuously modify the direction of their cursor path to reach the target (see Figure 1). The specific control-to-display misalignments were selected from a set of rotations about general threedimensional axes that varied in magnitude between 0 and 70 degrees, potentially making the targeting task very difficult. When the task difficulty was measured in terms of normalized path length, it was found that the task was approximately 20 times harder than the 2-D version of the task reported previously [23].

Subjects in the post-test only group completed the identical sequence of balloon popping tasks to those in the pre/post-test group, but were given the SSQ only after they had completed the targeting sequence ${ }^{1}$. The task took about 25 minutes to complete resulting in about 30 minutes of total immersion in the virtual environment.

\section{Results.}

Previous research has treated SSQ ratings as interval data [24]. We therefore used parametric statistics for our primary analysis, but also checked our results with nonparametric Mann-Whitney U tests. Since in some cases the independent groups in our comparison had significantly different variances, we used t-tests for independent groups, assuming unequal variances.

\footnotetext{
${ }^{1}$ Task analysis of subject performance on this 3D task has not yet been finalized and will not be reported in this paper.
}

The principal hypothesis of the experiment is tested by a comparison of the SSQ score from the post-test of the post-test only group compared to the post-test of the group that also had a SSQ pre-test. Figure 2 shows that the pre-test indeed caused approximately an $80 \%$ increase in the mean SSQ score. This difference is statistically reliable passing a t-test assuming unequal variance $(t(28)=2.6 p<0.03$ one-tailed $)$ and checked with Mann-Whitney $\mathrm{U}^{2},(U=69.5, p<0.038$ one-tailed $)$. One-tailed tests are used here because of the clear directional hypothesis. The assumption of equal variance was not used because of the clear difference in variance between the groups verified by an F-test $(F(13,15)=$ $5.41, p<0.003$ ). Figure 3 shows the post-test Nausea component of the post-test only group compared with the Nausea component from the post-test of the pre/post-test group. This comparison also shows the hypothesized effect in that the pre-testing increases the score of the subsequent post-test in the pre/post group. This difference is also statistically reliable $(t(28)=2.467, p<$ 0.020); Mann-Whitney $U=70.5, p<0.043$ )

It is also interesting to determine whether immersion in the virtual environment itself caused subjects to report SSQ symptoms. This may be tested by comparing the pre-test SSQ score of the pre/post test group with the post-test of the post-test only group. For this comparison, the pre/post group provides a baseline SSQ for our unexposed population with which the post-test only SSQ may be compared. Because we did not have a clear directional hypothesis for this comparison, and indeed because recently we have had almost no spontaneous reports of motion sickness in our virtual environment, we felt two-tailed tests more appropriate. Both a t-test and a Mann-Whitney U test show that the difference due to immersion itself is statistically significant $(t(27)=2.58, p<0.02 ; U=42, p<0.01)\left(\right.$ Figure 4). ${ }^{3}$ As was done for the principle comparison reported above, we also isolated the Nausea component of the SSQ for this comparison. This result is shown in Figure 5 and is also statistically significant $(t(27)=2.27, p<0.03 ; U=54, p<0.03)$.

\section{Discussion.}

The data in Figures 2 through 5 allow comparison of the changes in the full SSQ score and the Nausea component. These comparisons confirm our principle hypothesis that the pre-test exposure to the questionnaire does indeed substantially increase the post-test SSQ score. In terms of the differences between the scores, the effect seems to have a larger influence on the Nausea component than the full SSQ score. Also, one can note that the effect of immersion itself on the Nausea score is much smaller than the effect of the pretesting with the questionnaire. In fact, the increase due to immersion is just about 1 unit. This is a small number compared to published results of nausea effects in virtual environments from studies also using the SSQ, e.g., [24] in which reports range up to 3.5 .

\footnotetext{
${ }^{2} U$ was not corrected for ties, making the test more conservative.

${ }^{3}$ One subject's pretest questionnaire was read but filled out ambiguously and therefore unusable. Her post-test questionnaire was, however, correctly filled out.
} 


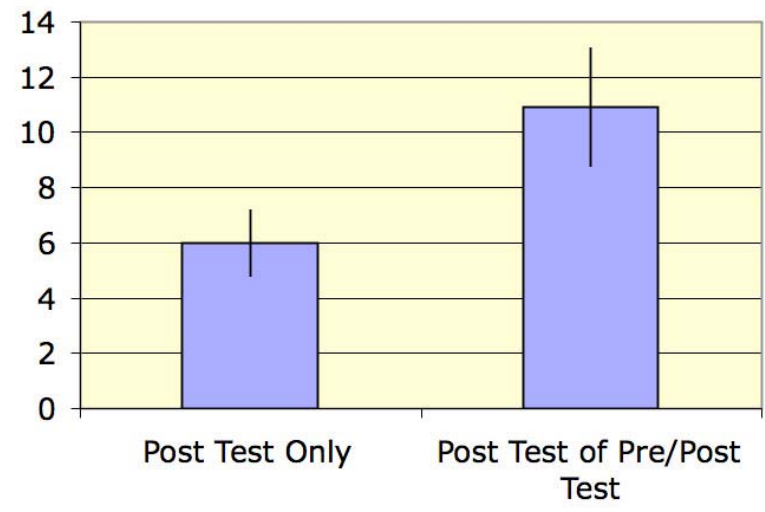

Figure 2. The effect of SSQ pre-testing on the SSQ scores. Error bars show $\pm 1 \mathrm{SE}$.

\section{Nausea}

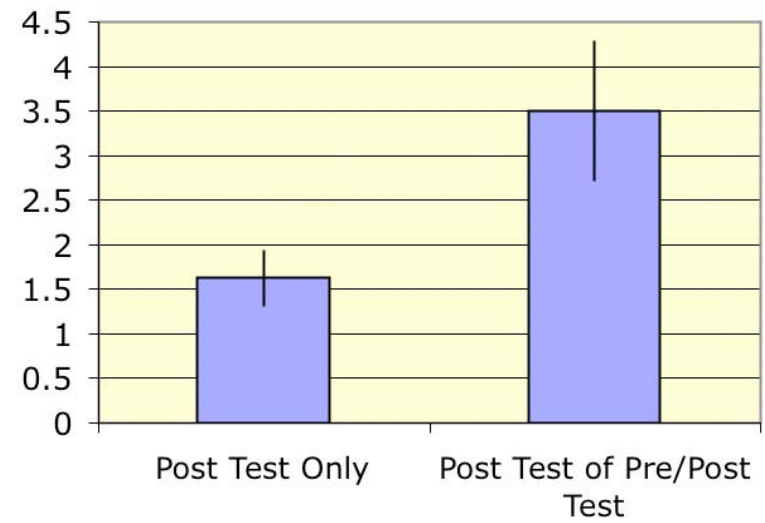

Figure 3. The effect of SSQ pre-testing on the Nausea component scores. Error bars show $\pm 1 \mathrm{SE}$.

The small increase of the Nausea scale we see due to immersion is consistent with our very infrequent reports of nausea in our virtual environment (system or this experiment?). Thus, our SSQ measurements imply that the suggestion of the possibility of motion sickness seems to have a greater impact than our environment and associated task themselves. Since our virtual environment may be unique because of its good spatial calibration, high rendering rate, and very low system latency, the relative difference we find between the effect of immersion and the effects of suggestion on simulation sickness may be different from other laboratories.

One problem in the current methods for measuring motion sickness is that it is a subjective, self-reported experience. It is difficult to avoid demand characteristics when a person is asked a question about how he or she is feeling both before and after an event. Instead, including physiological or objective measures with the subjective scales may increase the objectivity of the data, reducing experimenter demand effects.

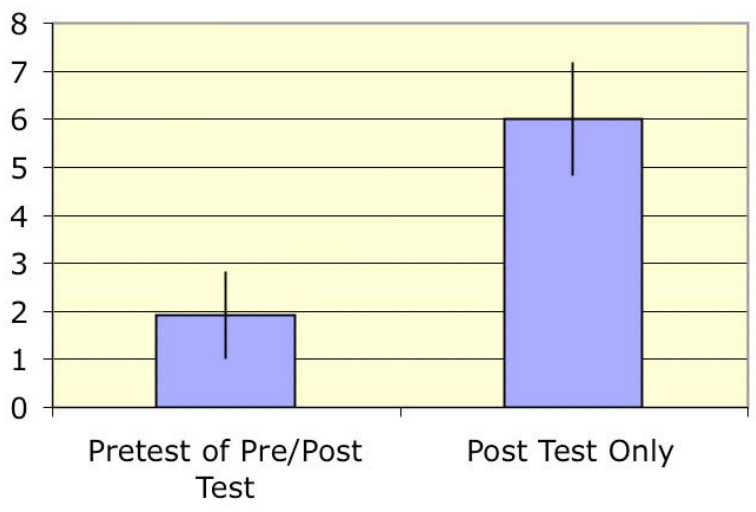

Figure 4. The effect of virtual environment immersion on SSQ scores. Error bars show $\pm 1 \mathrm{SE}$

Nausea

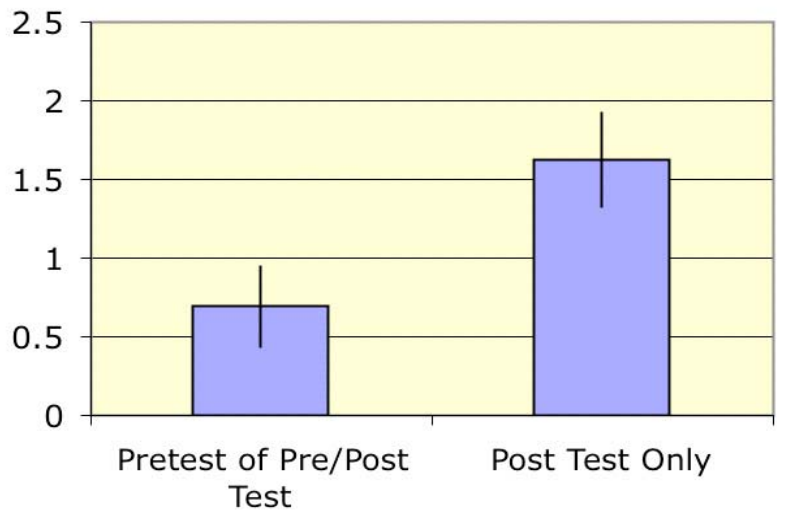

Figure 5. The effect of virtual environment immersion on the Nausea component of the SSQ. Error bars show \pm 1 SE.

While we have shown that demand characteristics exist, and reports of motion sickness can be influenced by questionnaire methods, we are by no means denying a relationship between virtual environments and motion sickness. In fact, when comparing the "pre" test of the pre/post test group with the post only group, we still find an effect for motion sickness. That is, immersion in the virtual environment in this study does appear to reflect reports of motion sickness, validating the self-reported experience of motion sickness. Therefore, although the reports of motion sickness appear valid, the methods employed in measuring motion sickness also contain demand characteristics that increase reported motion sickness. In fact, administering a pre-immersion SSQ appears to increase reported motion sickness by about $80 \%$ if treated as a ratio scale. One conclusion we may draw is that SSQ reports of the prevalence of motion sickness as measured by pre/post exposure questionnaire techniques may substantially overestimate its incidence. This conclusion may well extend to other environments in which motion sickness is assessed by pre/post exposure questionnaires. 
Our demonstration that pretesting with a SSQ can exaggerate reports of motion sickness confirms that the manner in which query information is presented matters. Indeed, it should be noted that the results of the current study could conceivably have been even stronger had the experimenter not merely mentioned the possibility of motion sickness when administrating the questionnaire but used stronger suggestions such as wearing a lab coat, obviously carrying nausea bags, and assuring the subjects that they would experience motion sickness during the task.

\subsection{Demand characteristics in other domains}

While we have focused on the effect that demand characteristics can have in reports of motion sickness in virtual environments, there are many other domains where demand characteristics play a role including surgical procedures, physical manipulations, prescribed diets, exercises, and other regimens [5],[6].

In the health domain, demand characteristics can have profound effects by leading the patient to expect a particular treatment result. In such cases the patient expects that the recommended treatment should and will produce a positive outcome [26]. There have been many reports of these "placebo effects" [27] where substances that are "given in the guise of active medication, but which in fact have no pharmacological effect on the condition being treated" [28].

These effects are typically explained in terms of an expectation that the treatment will produce the intended effects [29], [30]. For example, pharmaceutical trials have repeatedly shown that a substantial amount of patients demonstrate improvement after being given a "fake" drug [26], [31], [32]. Placebo effects and demand characteristics therefore need to be taken into account in experimental evaluations so that positive changes are not wrongly attributed to a treatment technology, when it was actually the situational demand that caused the improvement. This caution, of course, applies just as strongly to evaluation of motion sickness countermeasures in virtual environments.

Alternatively, and more central to our research, nocebo effects [33] have been observed in patients. In contrast to a placebo effect, a nocebo effect occurs when the demand characteristic creates a negative outcome. For example, in a study on the side effects of aspirin, subjects who were warned of aspirin's gastrointestinal side effects were almost three times more likely to report experience of these adverse events than patients who were not forewarned [34]. The expectations or demand characteristics influenced the patient to report experiencing the side effects. Nocebo effects are important and related to the present studies. The current pre/post-testing methods for measuring motion sickness in a virtual environment can exaggerate self-reports of motion sickness experienced during virtual environment use. Just as nocebo effects lead a person to think that negative symptoms will result, the pre-questionnaire indicates that a person immersed in a virtual environment is more likely to experience motion sickness.

\subsection{Conclusions}

Measurements of motion sickness by pre and post self-report questionnaires are significantly biased by demand characteristics. Comparative studies of motion sickness in virtual environments should employ experimental designs not subject to such biases or at least take measures to balance the biases. Alternatively, more objective physiological measures may be used with awareness that these measures too can be subject to experimental demand characteristics as noted in the Introduction.

Early worries that motion sickness could significantly limit that application of virtual environment technologies [35] clearly have been shown by experience to have some basis, but motion sickness in a fast, low-latency, well-calibrated system for manipulative simulation does not appear to be a major problem when the experimental demand characteristics are controlled.

It is important to note in conclusion that our finding that pretest questionnaires can inflate measures of motion sickness taken in virtual environments does not rule out questionnaire measurement techniques. Researchers need only determine the baseline questionnaire statistics for their subject population. Results from post-test only motion sickness evaluations may then be compared to these norms. Power analyses [36] may be used to determine the number of subjects needed to detect differences from these norms. In practical terms this approach is, however, likely to increase the number of subjects needed. It also risks distortions caused by inclusion of subjects who might exhibit motion sickness symptoms due to illness.

Consequently, all subjects should be screened for absence of illnesses. To reduce the possibility that the screening could be suggestive of motion sickness effects, it should be done in the context of admission to a general subject pool rather than as a requirement for a specific experiment. This type of generic prescreening may also be used to inform subjects of the overall possibility of motion sickness without specifically suggesting that a particular experiment might provoke it.

Finally, it should be noted that though physiological measurement techniques can provide a potentially more objective motion sickness measure, these also need to be compared to norms since they too can be influenced by suggestion [37].

\section{Acknowledgments.}

This work was supported by a NASA Space Human Factors grant titled "Virtual environment interfaces for remote operations" to Stephen R. Ellis and Bernard D. Adelstein. The authors also wish to thank Drs. Patricia Cowings and William Toscono for guidance and advice regarding motion sickness.

\section{References}

[1] M. T. Orne, "On the social psychology of the psychological experiment: With particular reference to demand characteristics and their implications," American Psychologist, Vol. 17, pp. 776-783, 1962.

[2] O. Pfungst, Clever Hans: The horse of Mr. Von Osten. New York: Holt, Rinehart, and Winston, 1965.

[3] R. Rosenthal, "On the social psychology of the psychological 
experiment: The experimenter's hypothesis as unintended determinant of experimental results," American Scientist, Vol. 51, pp. 268-283, 1963.

[4] J. W. Kanter, R. J. Kohlenberg, and E. F. Loftus, "Demand Characteristics, Treatment Rationales, and Cognitive Therapy for Depression," Prevention and Treatment, Vol. 5, p. 41, 2002.

[5] A. K. Shapiro. "A Contribution to A History of the Placebo Effect." Behaviourial Science, Vol. 5, pp. 109-135, 1960.

[6] A. K. Shapiro and E. Shapiro. "Patient-provider relationship and placebo effect," in Behavioral Health: A Handbook of Health Enhancement and Disease Prevention, J.D. Mattarazzo, S.M. Weiss, J.A., Herd, and N.E. Miller, Eds. New York: Wiley-Interscience, 1984, pp. 371-383.

[7] H. S. Friedman, Health Psychology, 2nd Edition. Upper Saddle River, New Jersey: Prentice Hall, 2002.

[8] J. D. Frank, Persuasion and Healing, Baltimore: Johns Hopkins Press, 1973.

[9] D. L. Paulhus, "Measurement and control of response bias," in Measures of Personality and Social Psychological Attitudes, Vol. 1, J. P. Robinson, P.R. Shaver, and L.S. Wrightsman, Eds. San Diego, CA: Academic Press, 1991, pp. 17-59.

[10] N. Schwartz, "Self reports: How the questions shape the answers," American Psychologist, Vol. 54, No. 2, pp. 93-105, 1999.

[11] S. R. Ellis, "What are virtual environments?," IEEE: Computer Graphics and Applications, Vol. 14, No. 1, pp. 17-22, 1994.

[12] P. DiZio, and J.R. Lackner, "Alleviation of motion sickness and postural instability during and after virtual environment exposure" (Technical Report). Prepared for Naval Air Warfare Center Training Systems Division, Orlando, FL, 1998.

[13] R. S. Kennedy, J. E. Fowlkes, and M. G. Lilienthal, M. G., "Postural and performance changes in Navy and Marine Corps pilots following flight simulators," Aviation, Space, and Environmental Medicine, Vol. 64, pp. 912-920, 1993.

[14] M. E. McCauley, and T.J. Sharkey, "Cybersickness: Perception of self-motion in virtual environments," Presence: Teleoperators and Virtual Environments, Vol. 1, No. 3, pp. 311-318, 1992.

[15] R. S. Kennedy, "Gaps in our knowledge about motion sickness and cybersickness," Paper presented at the Motion Sickness, Simulator Sickness, Balance Disorders and Sopite Syndrome Conference, September 9-11, New Orleans, LA, 1988.

[16] R. S. Kennedy, K. M. Stanney, J. M. Drexler, D. E. Compton, and M.B. Jones, "Computerized methods to evaluate virtual environment aftereffects," in Proceedings of the Driving Simulation Conference "DSC'99". Paris, France: French Ministry of Equipment, Transport, and Housing, 1999, pp. 273-287.

[17] R. S. Kennedy, N.E. Lane, K. S. Berbaum, and M. G. Lilienthal. "Simulator sickness questionnaire: an enhanced method for quantifying simulator sickness," International Journal of Aviation Psychology, Vol. 3, No. 3, pp. 203-220, 1993.

[18] R. S. Kennedy, and J. E. Fowlkes, "Simulator sickness is polygenic and polysymptomatic: Implications for research," International Journal of Aviation Psychology", Vol. 2, No. 1, pp. 23-38, 1992.

[19] R. S. Kennedy, J. M. Drexler, and K. S. Berbaum, "Methodological and measurement issues for identification of engineering features contributing to virtual reality sickness," in Proceedings of IMAGE VII, pp. 245-254, 1994.
[20] S. Nichols, S. Cobb and J. R. Wilson. Health and safety implications of virtual environments: measurement issues," Presence: Teleoperators and Virtual Environments, Vol. 6, No. 6, pp. 667-675, 1997.

[21] S. R. Ellis, B. D Adelstein, S. Baumeler, G. J. Jense, and R. H. Jacoby, "Sensor Spatial Distortion, Visual Latency, and Update Rate Effects on 3D Tracking in Virtual Environments," in Proceedings, IEEE VR '99, Houston TX, pp. 134$139,1999$.

[22] M. I. Hill, B. D. Adelstein, and S. R. Ellis, "Achieving minimum latency in virtual environment applications" in Proceedings of IMAGE Society Meeting, 2004. http://www.public.asu.edu/ image/PUBs/Publications.html

[23] S. R. Ellis, B. D. Adelstein, and R.B. Welch, "Kinesthetic compensation for rotational sensorimotor rearrangements: demonstration with experimental controls," in Proceedings of HFES, pp. 1551-1556, 2002.

[24] R. H. So, W. T. Lo, and A. T. K. Ho, "Effects of navigation speed on motion sickness caused by an immersive virtual environment," Human Factors, Vol. 43, No. 3, pp. 452-461, 2001.

[25] S. D. Young and D. Oppenheimer, "Framing Matters," Clinical Therapeutics, under review.

[26] R. Crow, H. Gage, S. Hampson, J. Hart, A. Kimber, and H. Thomas, "The role of expectancies in the placebo effect and their use in the delivery of health care: a systematic review," Health Technology Assessment, Vol. 3, No. 3, pp. 1-96, 1999.

[27] K. Beecher, "The powerful placebo," Journal of the American Medical Association. Vol. 159, pp. 1602-1606, 1955.

[28] I. Kirsch, "Response expectancy as a determinant of experience and behavior," American Psychologist, Vol. 40, pp. 1189-1202, 1985.

[29] I. Kirsch, "The placebo effect as a conditioned response: Failures of the "litmus test,"' Behavioral and Brain Sciences, Vol. 14, pp. 200-204, 1991.

[30] I. Kirsch, "Specifying nonspecifics: Psychological mechanisms of placebo effects," in The Placebo Effect: An Interdisciplinary Exploration, A. Harrington, Ed. Cambridge, MA: Harvard University Press, pp. 166-186, 1997.

[31] K. L. White, The Task of Medicine: Dialogue at Wickenbug. Menlo Park, CA: The Henry J. Kaiser Family Foundation, 1988.

[32] D .E. Moerman, and B. J. Wayne, "Deconstructing the placebo effect and finding the meaning response," Annals of Internal Medicine, Vol. 136, No. 6, pp. 471-6, 2002.

[33] R. A. Hahn, "The nocebo phenomenon: Scope and foundations," in The Placebo Effect: An Interdisciplinary Exploration, A. Harrington, Ed. Cambridge, MA: Harvard University Press, pp. 56-76, 1997.

[34] M. G. Myers, J. A. Cairns, and J. Singer, "The consent form as a possible cause of side effects," Clin Pharmacol Ther, Vol. 42, pp. 250-253, 1987.

[35] Multiple articles in section: Spotlight on Simulator Sickness, Presence: Teleoperators and Virtual Environments, Vol. 1, No. 2, pp. 295-363, 1992.

[36] J. Cohen. Statistical Power Analysis for the Behavioural Sciences. Academic Press: New York, 1969.

[37] J. T. Reason and J. J. Brand. Motion Sickness. London: Academic Press, 1975. 